\title{
Highly Stable Photonic Local Carriers for Phased Array Receiver System
}

\author{
Md. Rezaul Hoque Khan \\ Islamic University of Technology, Dhaka, Bangladesh and \\ University of Twente, Netherlands \\ rhkhan@iut-dhaka.edu
}

\author{
Md. Ashraful Hoque \\ Islamic University of Technology \\ Dhaka, Bangladesh \\ mahoque@iut-dhaka.edu
}

\begin{abstract}
In this paper, a complete system analysis of photonic local carrier generation technique has been investigated. The generated carrier is potentially suitable to replace the existing microwave/RF Local Carrier (LC) used in commercial Low Noise Blocks (LNBs) for the Phased Array (PA) receiver system. The optical LC generated from heterodyning of two commercialized lasers is being stabilized with an Optical Frequency Lock Loop (OFLL). This approach resulted in a generated carrier at the $K_{u^{-}}$ band $(10.7 \mathrm{GHz}$ to $12.75 \mathrm{GHz})$ signal received from a $\mathrm{PA}$ receiver. Various loop parameters of the OFLL have been investigated to comply with the requirements of the commercial LNBs The proposed OFLL shows a 2400 fold improvement in the frequency stability at 1000 s averaging time compared to its free running condition. It is also demonstrated that with an optimized loop gain of $30 \mathrm{~dB}$, the loop response time of the proposed OFLL becomes $11 \mu \mathrm{s}$.
\end{abstract}

Keywords-Optical Frequency Lock Loop (OFLL); microwave carrier generation; locking range; frequency stability

\section{INTRODUCTION}

Stabilization of laser frequency differences is essential in many modern experimental schemes. Applications extend from advanced optical fiber telecommunications in atomic clocks or in high resolution atomic and molecular spectroscopy [1-4] to precision spectroscopy and sensing $[5,6]$. Phase coherence of the two laser fields locked at a frequency offset is not required in many applications and a mere frequency lock is an adequate solution. In any case, the Optical Phase Lock Loop (OPLL) is a standout amongst the most commonly utilized locking techniques. When the OPLL is designed to ensure only the stabilization of the frequency drift and not the consistency of the phase, it is called Optical Frequency Locked Loop (OFLL). In all these applications, a precisely defined optical frequency is needed and long-term stability must be ensured for correct system operation, with a required degree of stability and accuracy that depends on the application. Practically speaking, a beat signal is produced between the laser to be frequencystabilized (slave) and a second laser with known frequency (master) [7]. To stabilize the optically generated carrier, the beat signal is often mixed down to a lower frequency which is far better to be taken care of electronically $[8,9]$. The main difference among the various frequency locking schemes which have been developed in the last decade, is the method used to generate and process the error signal employed for locking the slave laser [10]. A very simple locking scheme was demonstrated in [11] using an electronic delay line in conjunction with a phase detector as a frequency dependent phase shifter. This scheme has the advantage of large capture range, but as a drawback the beat frequency must be tuned by a manual adjustment of the delay line length. As a consequence, real-time, rapid tuning of the beat frequency is impossible. Other methods used to generate beat frequency use frequency multiplier. A different approach converts the beat frequency to a proportional voltage by a Frequency-to-Voltage Converter (FVC). The voltage is then compared to a reference voltage, which sets the beat frequency [12]. This approach is limited to the maximum operating frequency of the commercial FVC and can be improved by using a hybrid analog-digital locking scheme using high performance FVCs [13]. Nevertheless the scheme suffers from the limited bandwidth of the FVC. Recently, a highly stable and wide tunable frequency locking scheme has been presented, where a high-speed frequency divider (prescaler) is used on the beat signal before processing the error signal [14]. The main disadvantage of the use of the prescaler is that it degrades the loop response time and limits the loop bandwidth. Generating the error signal from the amplitude response of an RF filter to realize a sensitive analog FVC is also proposed [8, 15]. An alternative of these modulation-free error conversion techniques is the use of electrical filters [16] or frequency multipliers [17].

However, to the best of our knowledge, none of the above schemes reported any investigation on the long term frequency stability of the beat spectrum of the OFLL. A simple OFLL technique based on the concept presented in [11] is proposed in this paper and its performance is improved with the use of a variable delay line in the frequency discriminator to facilitate beat frequency tuning.

\section{PROPOSED OPTICAL FREQUENCY LOCKED LOOP SCHEME}

The frequency of a diode laser depends on the injection current and the temperature and is very sensitive to fluctuations of those parameters. For example, the DFB laser (Avanex inc., A1905LMI) used in our experiment has a frequency sensitivity to injection current and temperature of $325 \mathrm{MHz} / \mathrm{mA}$ and $10 \mathrm{GHz} /{ }^{\circ} \mathrm{C}$ respectively [18]. Several studies and experiments show that the beat signal generated by a free-running heterodyning system suffers a substantial frequency drift [19]. Laser frequency drifts by hundreds of $\mathrm{MHz}$ in an ordinary 
environment [20]. The maximum permissible frequency drift of the generated carrier is $5 \mathrm{MHz}$ [21], according to the DVB-S specification [22]. The carrier signal, generated by heterodyning of two lasers, is used for the downconversion of the $10.7 \mathrm{GHz}$ to $12.75 \mathrm{GHz} \mathrm{K}_{\mathrm{u}}$-band signal received from a Phased Array Antenna (PAA). The block diagram of the proposed OFLL is presented in Figure 1. The proposed scheme is able to generate an adjustable carrier as specified in [22].

(a)

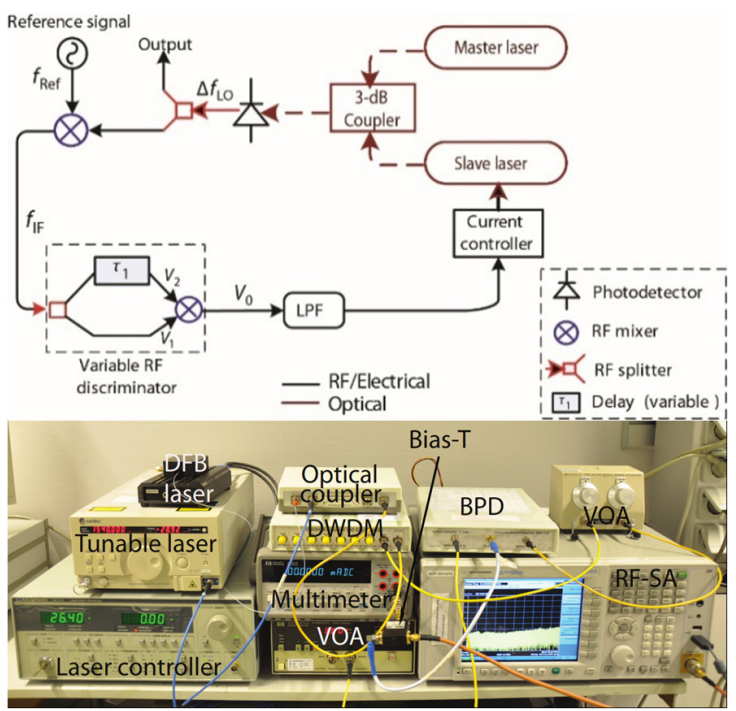

Fig. 1. Proposed OFLL: (a) block diagram, (b) experimental setup.

The two lasers used for the experiments are a DFB laser Avanex A1905LMI and a Tunable Laser Diode (TLD) Santec TSL-210. In the experiment, the tunable laser and the DFB laser are employed as a master laser and slave laser having optical frequencies $f_{M L}$ and $f_{S L}$, respectively, to produce the beat frequency, $\Delta f_{L O}=f_{M L}-f_{S L}$. The beat signal is provided by a $20 \mathrm{GHz}$ bandwidth photodetector (Discovery Semiconductor DSC30S) and is amplified by $20 \mathrm{~dB}$ by a commercial RF amplifier (MiniCircuit ZX60-183). A power splitter (MiniCircuit ZFRSC-123-S) is used to tap the beat signal for monitoring. The output signal from the power splitter is mixed with a reference signal, $f_{\text {Ref }}$, at $\sim 10 \mathrm{GHz}$ frequency provided by a signal generator (Agilent PSG E8267D). An error frequency, $f_{e}=\Delta f_{L O}-f_{R e f}$, is produced at the output of the RF mixer (MiniCircuit ZX05-153LH-S). The error signal, $V_{e} \cos \left(2 \pi f_{e} t\right)$, is then passed through a variable frequency discriminator, where $V_{e}$ is the amplitude of the error signal. In the frequency discriminator, the signal is divided into two equal parts by an RF splitter and recombined at a mixer (MiniCircuits SBL 48) after a variable delay line (Narda 3752) has deferred one part. Hence, we can write:

$$
\begin{gathered}
V_{1}=\frac{V_{\mathrm{e}}}{2}\left[\cos \left(\omega_{\mathrm{e}} \mathrm{t}\right)\right] \\
V_{2}=\frac{V_{\mathrm{e}}}{2}\left[\cos \left(\omega_{\mathrm{e}}\left(t-\tau_{1}\right)\right)\right]
\end{gathered}
$$

where $V_{1}$ and $V_{2}$ are the signals combined in the mixer, $\omega_{e}=2 \pi\left(\Delta f_{L O}-f_{R e f}\right)$, and $\tau_{1}$ is the time delay. The resultant signal of the frequency discriminator at the output of the mixer, $V_{0}$ is [23]:

$$
V_{0}=\frac{V_{\mathrm{IF}}}{\pi}\left[\cos \left(2 \pi f_{\mathrm{IF}} \tau_{1}\right)+\cos \left(2 \pi f_{\mathrm{IF}}\left(2 t-\tau_{1}\right)\right)+\ldots\right]
$$

The high-frequency term (the $2^{\text {nd }}$ term in the bracket) is eliminated by a Low Pass Filter (LPF) (MiniCircuit SLP-50), called the loop filter. As a result, the LPF output produces a series of nulls when $\omega_{e} \tau_{1}=(2 n+1) \pi / 2$, where $n=$ $\pm 0, \pm 1, \pm 2 \ldots$ The feedback system acts on the slave laser injection current and allows to actively control the emission frequency of the slave laser, so that a constant frequency difference between the slave and the master laser is maintained. The frequency of the slave laser is tuned by applying an external voltage to its injection current controller (ILX Lightwave LDC-3724). The conversion factor of this external voltage and the change of emission frequency of the slave laser, $K_{S L}$, is measured to be $31.25 \times 10^{-2} \mathrm{MHz} / \mathrm{mV}$.

\section{A. Characterization of the RF Discriminator}

Utilizing a passive RF component and realizing a delay of $\tau_{1}=3 \mathrm{~ns}$, the discriminator output at the LPF as a function of the beat frequency is shown in Figure 2. The offset frequency between the first null from the reference frequency is given by $\Delta f=1 / 2 \tau_{1}$, where $1 / \tau_{1}$ denotes the spacing of the nulls that the OFLL lockes on. The point A and B in Figure 2 is the nearest null to the reference frequency where the frequency to voltage conversion factor, $K_{d}$, is maximum and amounts to be $300 \mathrm{mV} / \mathrm{GHz}$. A higher $\tau_{1}$ will reduce the $\Delta f$ and will increase the slope at the nulls which ultimately will give a higher conversion of $K_{d}$. The various parameters and their values used in our analysis are summarized in Table I.

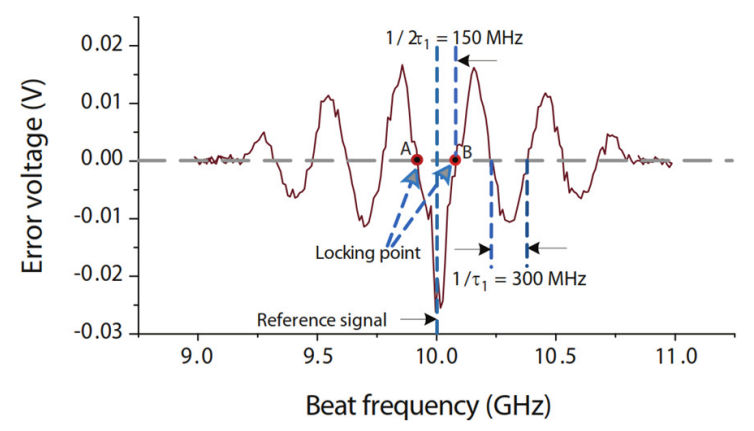

Fig. 2. Beat frequency as a function of error voltage for a delay of $\tau_{1}=3 \mathrm{~ns}$.

TABLE I. VARIOUS LOOP PARAMETERS

\begin{tabular}{|c|c|c|}
\hline Parameter & Value & Dimension \\
\hline Total gain of the RF amplifiers & $30 \mathrm{~dB}$ & $K_{a}$ \\
\hline External voltage-to-frequency & $31.25 \times 10^{-2}$ & $K_{S L}$ \\
\hline Conversion factor of the slave laser & $\mathrm{MHz} / \mathrm{mV}$ & \\
\hline Frequency-to-voltage conversion & $300 \mathrm{mV} / \mathrm{GHz}$ & $K_{d}$ \\
\hline Factor of the discriminator & & \\
\hline Time constant of the current & $300 \mu \mathrm{s}$ & $\tau_{0}$ \\
\hline Controller of the slave laser & & \\
\hline Time constant of the LPF & $1.58 \mu \mathrm{s}$ & $\tau_{2}$ \\
\hline
\end{tabular}




\section{OPTICAL FREQUENCY LOCK LOOP ANALYSIS}

The proposed OFLL in Figure 1 can be represented as a generic model for the feedback system and its s-domain representation is presented in Figure 3. In this model of the OFLL, the optical and the electrical connections are represented by doted and solid lines respectively. The optical field from the master and the slave laser are represented by $E_{L 1}$ and $E_{L 2}$ respectively. The fields are combined and put to a PD. The beat frequency is mixed with the reference frequency, $f_{\text {Ref }}$. The downconverted signal is then amplified by an RF amplifier having a gain of $K_{a}$. An RF frequency discriminator having a frequency-to-voltage conversion factor $K_{d}$ converts the downconverted frequency into proportional error voltage. The error signal is passed through a LPF having a Laplace transform of the frequency response, $F(s)$, to filter out the high frequency component from the error signal. The error signal is applied to the current controller of the slave laser. The external voltage-to-frequency conversion factor and the time constant of the slave laser controller are denoted by $K_{S L}$ and $\tau_{0}$ respectively.

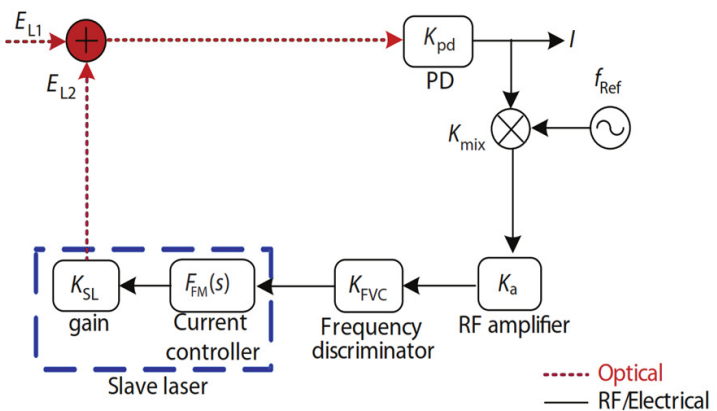

Fig. 3. Linearized s-domain representation of the OFLL.

From the linearized s-domain representation of the OFLL the open-loop transfer function of the OFLL is defined as the product of the transfer functions of all the elements in the loop:

$$
G(s)=K_{p d} K_{m i x} K_{a} K_{F V C} K_{S L} F_{F M}(s)=K F_{F M}(s)
$$

where $K=K_{p d} K_{m i x} K_{a} K_{F V C} K_{S L}$ is the total gain. The closedloop transfer function of the OFLL is [24]:

$$
\begin{aligned}
H(s) & =\frac{K 4 \pi^{2} f_{r}^{2}}{s^{2}+s 2 \pi f_{c}+4 \pi^{2} f_{r}^{2}(1+K)} \\
& =\frac{4 \pi^{2} \mathrm{f}_{\mathrm{n}}{ }^{2}}{\mathrm{~s}^{2}+\mathrm{s} \zeta 2 \pi \mathrm{f}_{\mathrm{n}}+4 \pi^{2} \mathrm{f}_{\mathrm{n}}{ }^{2}\left(1+\frac{1}{\mathrm{~K}}\right)}
\end{aligned}
$$

where $f_{n}$ and $\zeta$ represent the natural frequency and the damping factor of the loop and are expressed by:

$$
\begin{gathered}
\zeta=\frac{f_{c}}{f_{n}} \\
f_{n}=f_{r} \sqrt{K}
\end{gathered}
$$

\section{EXPERIMENTAL RESULTS}

In this section we will the stability of the OFLL will be investigated and analyzed. Moreover, some other functionalities of a carrier, namely the maximum tuning range and the maximum tuning rate (the speed at which the generated carrier can be tuned to a certain frequency) need to be investigated. Like any feedback system, the dynamics of these functionalities is determined by various parameters like loop gain and loop response. Also, the effect of various loop parameters (i.e. loop natural frequency and damping factor) on these functionalities of the OFLL will be investigated.

\section{A. Locking Offset Range and Capture Range}

The discriminator signal at the output of the LPF as a function of the beat frequency is shown in Figure 3. The error voltage is applied to the external tuning port of the current controller of the slave laser. This will ultimately change the emission frequency of the slave laser until the error voltage becomes zero. Hence, the generated carrier signal is eventually locked to the reference signal with a fixed offset frequency. The relation of the delay in the frequency discriminator and the offset frequency, as given in (4) is plotted in Figure 4. The measured values are also plotted and found to be close to the calculated values.



Fig. 4. The relation of the delay in the frequency discriminator and the offset frequency voltage for a delay of $\tau_{1}=3$ ns.

From Figure 3, with $\tau_{1}=3.3 \mathrm{~ns}$, the locking $150 \mathrm{MHz}$ offset frequency from the reference signal and the nulls are spaced by $300 \mathrm{MHz}$. Figure 4 shows the relation of the delay in the frequency discriminator and the offset frequency. This offset frequency range also gives the range of frequencies from the reference signal at which the free-running carrier signal becomes locked, also called the capture range of the OFLL. In Figure 3 , the error signal provides a capture range given by $\pm 1 / 2 \tau_{1}= \pm 150 \mathrm{MHz}$

\section{B. Beat Frequency Tuning}

Once the optically generated carrier signal is locked with the reference signal, the generated carrier signal can be tuned by simply varying the delay. The beat frequency is locked at $125,135,142,151$ and $166.6 \mathrm{MHz}$ offset frequency from the reference frequency. As given in (4), for delays of 3, 3.3, 3.5, 3.7 , and $4 \mathrm{~ns}$ the beat frequency will be locked at 166.6, 151, 142,135 , and $125 \mathrm{MHz}$ respectively. The beat frequency tuning for various delays is shown in Figure 5. 


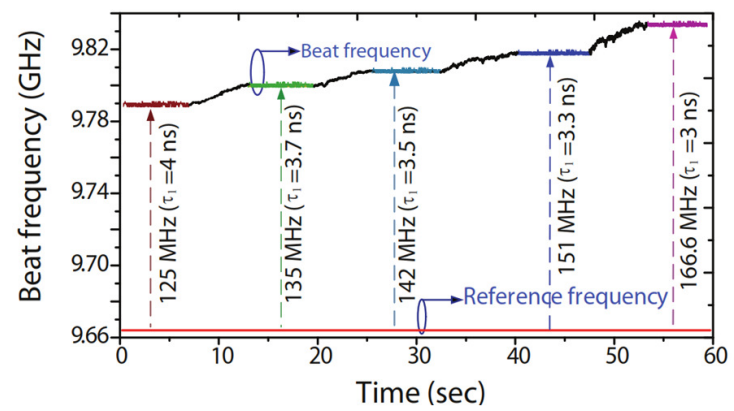

Fig. 5. Beat frequency tuning by varying delay.

\section{Frequency Resolution}

The frequency resolution is determined by the slope of the error signal at the locking point. As indicated by (4), a longer delay line $\tau_{1}$ reduces the capture range but enhances resolution. In order to demonstrate the effect of delay on the sensitivity, for a fixed delay, the reference frequency was tuned from $9.66 \mathrm{GHz}$ to $9.54 \mathrm{GHz}$, as shown in Figure 6. Depending on the delay, for example $3,3.5$, and $4 \mathrm{~ns}$, the beat frequency is also tuned with an offset frequency of $166.6,142$, and $125 \mathrm{MHz}$ respectively from the reference frequency. Figure 6 shows the frequency resolution of the locked signal for various delays. In the measurement the reference frequency was changed to demonstrate the frequency resolution. Moreover, the inset in Figure 6 shows the change of resolution due to the change of delay.

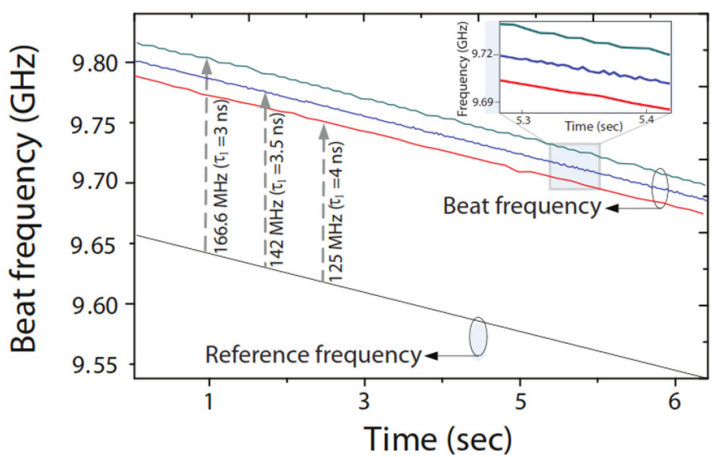

Fig. 6. Frequency resolution of the locked signal for various delays. The inset shows a highlighted portion of the measurement.

\section{Loop Response Time}

The step response provides an insight in loop response time and loop settling time. Note that the time taken for a feedback system to stay within $10 \%$ of its final value is called settling time. One widely used measure of the response speed of a feedback system is the time it requires to reach the $90 \%$ of its final value, which is called loop response time. With the help of (6) and (7), for a given loop bandwidth $(1 \mathrm{MHz}$ in our experiment), the calculated loop response time values are plotted in Figure 7 for various loop natural frequencies and damping factors. From Figure 7 we can see that for a $1 \mathrm{MHz}$ loop bandwidth, the loop response becomes faster for a lower damping factor with higher settling time. The optimum value of the damping factor should be chosen by carefully considering both loop response time and settling time. From Figure 7 we can observe that there is a trade-off between the loop response time and settling time. With higher damping factor, the loop response time increases with decreasing settling time. The optimum value of the damping factor can be indicated as $\zeta=1$ where both loop response time and settling time are optimum. The optimized loop natural frequency $f_{n}$ is $1 \mathrm{MHz}$.

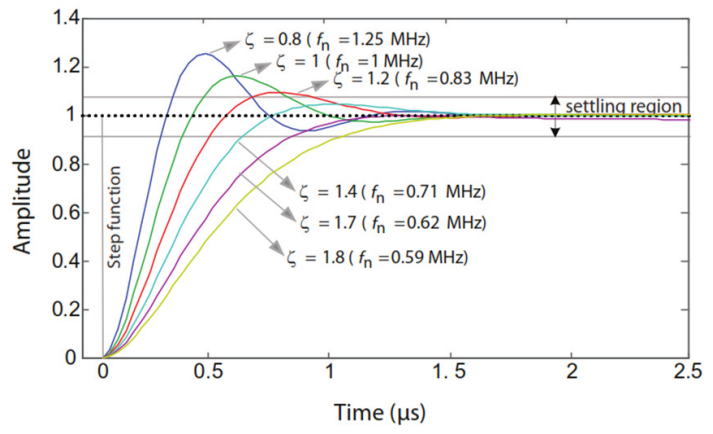

Fig. 7. Loop response time for various natural frequencies and damping factors.

The step response measurement procedure is shown in Figure 8. The step signal from a vector signal generator (Agilent PSG E8267D) will introduce an external disturbance in the current controller of the slave laser (DFB laser) of the OFLL and due to this aggravation, the signal will suffer a temporary deviation from the desired frequency. The OFLL will recover the system into the former stable condition soon after the disturbance and this duration of the generated carrier signal from its unstable condition is measured by an oscilloscope (Agilent infinium 54854A) by synchronizing with the vector signal generator through an external trigger. Employing the values of the parameters in Table I, for a given value of gain, $K_{a}=14 \mathrm{~dB}$, the natural frequency, $f_{n}$, and the damping factor, $\zeta$, can be calculated using (7) and (8) as $1 \mathrm{MHz}$ and 1 , respectively. For $K_{a}=10 \mathrm{~dB}$ the calculated values of the natural frequency and the damping factor become $f_{n}=0.62 \mathrm{MHz}$ and $\zeta=1.7$ respectively.

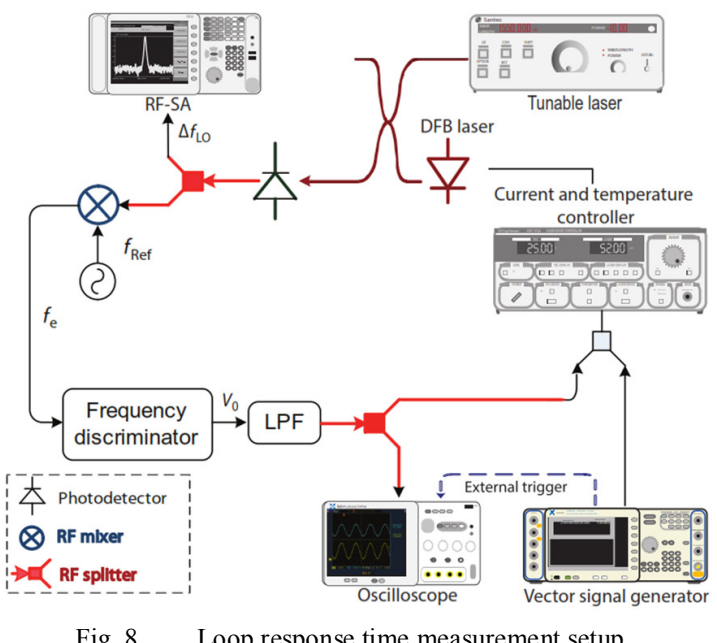

Fig. 8. Loop response time measurement setup. 
From Figure 9, for $\zeta=1$ and $\zeta=1.7$ the speed of the response of the loop is found to be 0.35 and $0.85 \mu$ s respectively. From Figure 9(a) it is evident that for a value of $\zeta=1$ the calculated loop settling time is found to be $0.78 \mu$ s which is slower than the $0.58 \mu \mathrm{s}$ (Figure 9(b)) for $\zeta=1.7$ (called critical damping). The experimental results are in good agreement with the calculated values. From Figure 9 we can observe that there is a trade-off between loop response time and settling time. With higher damping factor, the loop response time increases with decrease of settling time. The optimum value of damping factor can be indicated as $\zeta=1$ where both loop response time and settling time are also optimum. The optimized loop natural frequency, $f_{n}$ is $1 \mathrm{MHz}$.

(a)



(b)



Fig. 9. Loop settling time comparison, using step response, on calculated and measured values for damping factor of (a) 1 and (b) 1.7 .

\section{E. Frequency Response}

The frequency response of the OFLL is plotted in Figure 10. The $\mathrm{x}$-axis has been normalized by dividing the frequency by loop natural frequency $f_{n}$. The plot shows how the loop behaves in the frequency domain. Figure 10 shows the result of the frequency response of the OFLL for various damping factors. The frequency response of the loop in Figure 10 looks very similar to that of the frequency response of a low pass filter. This is what an OFLL acts in practice. If the frequency is equal to the natural frequency (i.e. when $f / f_{n}=1$ ) the oscillation becomes very large. This phenomenon is reflected in Figure 10 (a) for $\zeta=1$. Larger damping factors have lower overshoot, i.e. better response, but also have long response time [29], as can be observed in Figure 10(b) for $\zeta=1.7$. Note that the higher frequency term in (3) needs to be filtered out, the response does it by keeping the loop bandwidth narrow. For the same values of natural frequency and damping factor, the calculated result of the frequency response is presented in Figure 10. The experimental results are in good agreement with the calculated values. The step/impulse response of the system, a time domain response, can be converted into a frequency domain response by simply performing Fourier transformation. The calculated frequency response of the loop in Figure 10 is actually a time domain response (step response/impulse) of Figure 7. The time and frequency domain measurement values from Figure 7 and Figure 10 are in a good agreement.

(a)
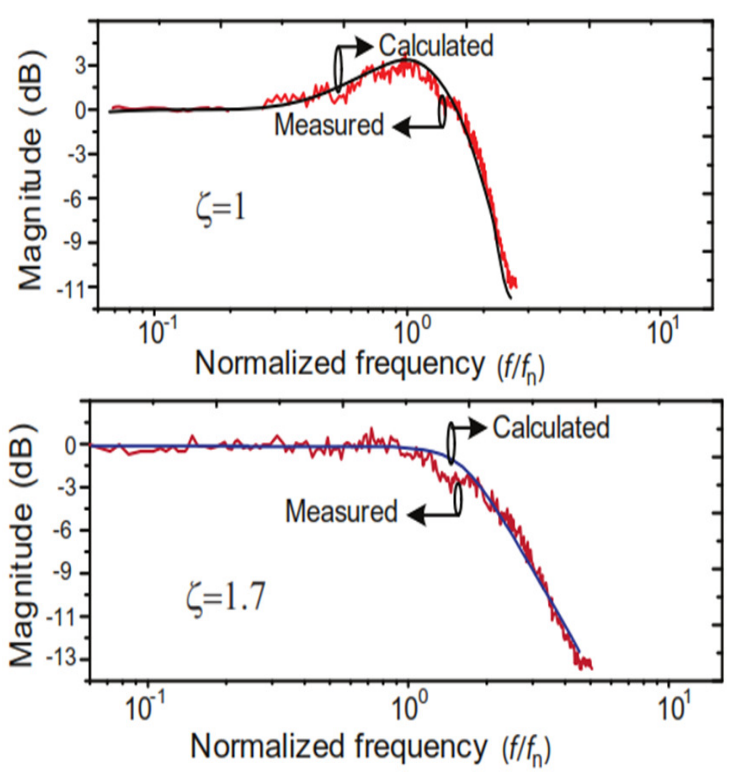

Fig. 10. Experimental and calculated results of the frequency response of the OFLL, the $x$-axis is the normalized frequency and the $y$-axis is the response in $\mathrm{dB}$.

\section{F. Long Term Frequency Stability Analysis}

Typically, a free-running microwave frequency, $f_{\text {beat }}$, deviates from the required frequency, $f_{\text {ideal }}$, with variable direction and rate of change. In order to express the long term frequency stability of a carrier, the Allan deviation $\sigma_{y}$ is often used. To evaluate this frequency stability, $\sigma_{y}$ is proven to be a valuable tool to quantify the stability of an optically generated microwave carrier [26]:

$$
\sigma_{y}^{2}(\tau)=\frac{1}{2(M-1)} \sum_{i=1}^{M-1} \llbracket \bar{y}_{i+1}-\bar{y}_{i}{ }^{2}
$$

where $y_{i}$ is the $i^{\text {th }}$ of the $M$ fractional frequency values averaged over the measurement (sampling) interval $\tau$. The measured frequency stability of the generated carrier is expressed in Allan deviation in Figure 11 for both locked and free running conditions. For the free-running condition, for an increased averaging time of $\tau=1 \mathrm{~s}$ to $\tau=1000 \mathrm{~s}$, the Allan deviation increases from $2.5 \times 10^{-10}$ to $6 \times 10^{-9}$. However, by implementing the OFLL the corresponding improved long-term frequency stability could be $3 \times 10^{-10}$ and $2.5 \times 10^{-12}$. The OFLL shows a 2400 -fold improvement in the frequency stability at 1000 s averaging time. A typical quartz oscillator (Wenzel 501- 
04623E) has an Allan deviation frequency stability of $4 \times 10^{-7}$ [27]. A typical atomic oscillator used as a clock source may provide a frequency stability of Allan deviation of $1 \times 10^{-10}$ for $1 \mathrm{~s}$ averaging time [28]. A radio frequency (RF) clock signal transmission over $100 \mathrm{~m}$ via fiber link results in an Allan deviation of $1.3 \times 10^{-10}$ for $1 \mathrm{~s}$ averaging time [29]. The long term frequency stability of the presented work shows the superior performance of Allan deviation of $1 \times 10^{-10}$ for an averaging time of $10^{3} \mathrm{~s}$ compared to OPLL setups involving integrated Phase-Frequency Detectors (PFD) [30].

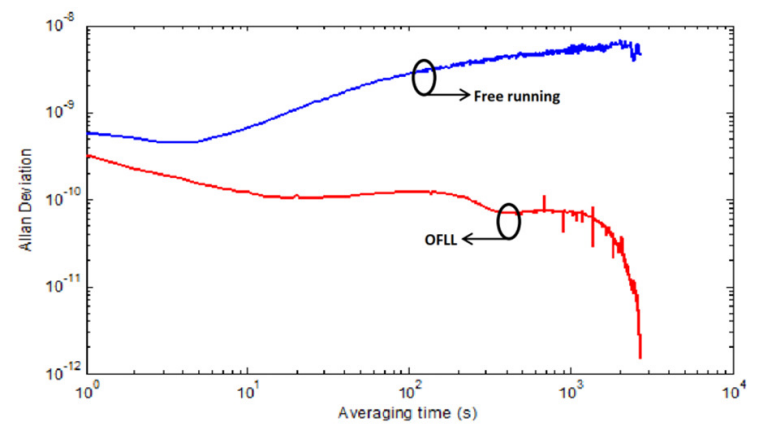

Fig. 11. Allan deviation of the frequency stability of the OFLL under freerunning and locked conditions.

\section{CONCLUSION}

System analysis and experimental demonstration of an optically generated LC designed to comply with the requirements of the standard LC signal used in commercial LNBs have been investigated in this paper. The LC signal was used for the downconversion of the $10.7 \mathrm{GHz}$ to $12.75 \mathrm{GHz}$ signal received from a PA receiver. An OFLL was also implemented to stabilize the generated LC signal. Detailed analysis of the OFLL scheme has been presented. The loop filter and the loop gain of the OFLL should be chosen properly to make the feedback system stable and fast. It was also demonstrated that with an optimized gain of $14 \mathrm{~dB}$, the loop response time becomes $0.35 \mu$ s with a settling time of $11.5 \mu \mathrm{s}$. The presented results emphasize the effectiveness of the use of the OFLL in improving the long-term stability of the freerunning microwave carrier by a 2400 -fold improvement in the frequency stability at 1000 s averaging time. Previous research merely presents straightforward time domain measurements and lacks complete frequency domain measurements of the loop response. The presented research focused on this highly interesting topic. Both time and frequency domain measurement values are in a good agreement with each other. Moreover, the presented implementation shows batter performance in terms of the capture range of $150 \mathrm{MHz}$ compared to the very recent investigation of a few hundred $\mathrm{kHz}$ in [31].

\section{ACKNOWLEDGMENTS}

The authors acknowledge the support of the Smart Mix Program of the Dutch Ministry of Economic Affairs and the Dutch Ministry of Education, Culture and Science.

\section{REFERENCES}

[1] S. H. Yim, S.-B. Lee, T. Y. Kwon, and S. E. Park, "Optical phase locking of two extended-cavity diode lasers with ultra-low phase noise for atom interferometry," Applied Physics B, vol. 115, no. 4, pp. 491495, Jun. 2014, doi: 10.1007/s00340-013-5629-5.

[2] M. Dąbrowski, R. Chrapkiewicz, and W. Wasilewski, "Hamiltonian design in readout from room-temperature Raman atomic memory," Optics Express, vol. 22, no. 21, pp. 26076-26091, Oct. 2014, doi: 10.1364/OE.22.026076.

[3] M. Parniak, A. Leszczyński, and W. Wasilewski, "Coupling of fourwave mixing and Raman scattering by ground-state atomic coherence," Physical Review A, vol. 93, no. 5, p. 053821, May 2016, doi: 10.1103/PhysRevA.93.053821.

[4] C.-H. Shin and M. Ohtsu, "Heterodyne optical phase-locked loop by confocal Fabry-Periot cavity coupled AlGaAs lasers," IEEE Photonics Technology Letters, vol. 2, no. 4, pp. 297-300, Apr. 1990, doi: $10.1109 / 68.53268$.

[5] M. Lyon and S. D. Bergeson, "Precision spectroscopy using a partially stabilized frequency comb," Applied Optics, vol. 53, no. 23, pp. 51635168, Aug. 2014, doi: 10.1364/AO.53.005163.

[6] R. Matthey, S. Schilt, D. Werner, C. Affolderbach, L. Thévenaz, and G. Mileti, "Diode laser frequency stabilisation for water-vapour differential absorption sensing," Applied Physics B, vol. 85, no. 2, pp. 477-485, Nov. 2006, doi: 10.1007/s00340-006-2358-z.

[7] M. R. H. Khan and M. A. Hoque, "A photonic frequency discriminator based laser linewidth estimation technique," International Journal of Advanced and Applied Sciences, vol. 6, no. 4, pp. 65-74, Apr. 2019, doi: 10.21833/ijaas.2019.04.008.

[8] G. Ritt, G. Cennini, C. Geckeler, and M. Weitz, "Laser frequency offset locking using a side of filter technique," Applied Physics B, vol. 79, no. 3, pp. 363-365, Aug. 2004, doi: 10.1007/s00340-004-1559-6.

[9] M. R. H. Khan, M. F. Islam, G. Sarowar, T. Reza, and M. A. Hoque, "Carrier generation using a dual-frequency distributed feedback waveguide laser for phased array antenna (PAA)," Journal of the European Optical Society-Rapid Publications, vol. 13, no. 1, p. 30, Oct. 2017, doi: 10.1186/s41476-017-0058-4.

[10] M. R. H. Khan et al., "Dual-Frequency Distributed Feedback Laser With Optical Frequency Locked Loop for Stable Microwave Signal Generation," IEEE Photonics Technology Letters, vol. 24, no. 16, pp. 1431-1433, Aug. 2012, doi: 10.1109/LPT.2012.2205379.

[11] U. Schünemann, H. Engler, R. Grimm, M. Weidemüller, and M. Zielonkowski, "Simple scheme for tunable frequency offset locking of two lasers," Review of Scientific Instruments, vol. 70, no. 1, pp. 242-243, Jan. 1999, doi: 10.1063/1.1149573.

[12] T. Stace, A. N. Luiten, and R. P. Kovacich, "Laser offset-frequency locking using a frequency-to-voltage converter," Measurement Science and Technology, vol. 9, no. 9, pp. 1635-1637, Sep. 1998, doi: 10.1088/0957-0233/9/9/038.

[13] J. Hughes and C. Fertig, "A widely tunable laser frequency offset lock with digital counting," Review of Scientific Instruments, vol. 79, no. 10, p. 103104 , Oct. 2008, doi: 10.1063/1.2999544.

[14] A. Castrillo, E. Fasci, G. Galzerano, G. Casa, P. Laporta, and L. Gianfrani, "Offset-frequency locking of extended-cavity diode lasers for precision spectroscopy of water at $138 \mu \mathrm{m}$," Optics Express, vol. 18, no. 21, pp. 21851-21860, Oct. 2010.

[15] N. Strauß, I. Ernsting, S. Schiller, A. Wicht, P. Huke, and R.-H. Rinkleff, "A simple scheme for precise relative frequency stabilization of lasers," Applied Physics B, vol. 88, no. 1, pp. 21-28, 2007.

[16] S. Schilt, R. Matthey, D. Kauffmann-Werner, C. Affolderbach, G. Mileti, and L. Thévenaz, "Laser offset-frequency locking up to $20 \mathrm{GHz}$ using a low-frequency electrical filter technique," Applied Optics, vol. 47, no. 24, pp. 4336-4344, Aug. 2008, doi: 10.1364/AO.47.004336.

[17] D. M. Perisic, A. C. Zoric, and Z. Gavric, "A Frequency Multiplier Based on Time Recursive Processing," Engineering, Technology \& Applied Science Research, vol. 7, no. 6, pp. 2104-2108, Dec. 2017.

[18] M. R. H. Khan, M. Burla, C. G. H. Roeloffzen, D. A. I. Marpaung, and W. van Etten, "Phase noise analysis of an $\mathrm{rf}$ local oscillator signal 
generated by optical heterodyning of two lasers," in 14th Annual Symposium of the IEEE Photonics Benelux Chapter, Brussels, Belgium, Nov. 2009, pp. 161-164.

[19] F. Friederich et al., "Phase-locking of the beat signal of two distributedfeedback diode lasers to oscillators working in the MHz to THz range," Optics Express, vol. 18, no. 8, pp. 8621-8629, Apr. 2010, doi: 10.1364/OE.18.008621.

[20] D.-H. Yang and Y.-Q. Wang, "Preliminary results of an optically pumped cesium beam frequency standard at Peking University," IEEE Transactions on Instrumentation and Measurement, vol. 40, no. 6, pp. 1000-1002, Dec. 1991, doi: 10.1109/19.119781.

[21] E. Casini, R. D. Gaudenzi, and A. Ginesi, "DVB-S2 modem algorithms design and performance over typical satellite channels," International Journal of Satellite Communications and Networking, vol. 22, no. 3, pp. 281-318, 2004, doi: 10.1002/sat.791.

[22] ETSI EN 301790 V1.5.1 (2009-05): Digital Video Broadcasting (DVB); Interaction channel for satellite distribution systems. Sophia Antipolis Cedex, France: ETSI, 2009.

[23] C. Toumazou, G. S. Moschytz, and B. Gilbert, Eds., Trade-Offs in Analog Circuit Design: The Designer's Companion. Springer US, 2002.

[24] D. R. Stephens, Phase-Locked Loops for Wireless Communications: Digital, Analog and Optical Implementations, 2nd ed. Springer US, 2002.

[25] F. M. Gardner, Phaselock Techniques, 3rd ed. USA: John Wiley \& Sons, 2005.

[26] H. Y. Ryu, S. H. Lee, and H. S. Suh, "Widely Tunable External Cavity Laser Diode Injection Locked to an Optical Frequency Comb," IEEE Photonics Technology Letters, vol. 22, no. 14, pp. 1066-1068, Jul. 2010, doi: 10.1109/LPT.2010.2049101.

[27] E. Rubiola, Phase Noise and Frequency Stability in Oscillators. Cambridge, UK: Cambridge University Press, 2008.

[28] S. Knappe et al., "Microfabricated atomic clocks and magnetometers," Journal of Optics A: Pure and Applied Optics, vol. 8, no. 7, pp. S318S322, May 2006, doi: 10.1088/1464-4258/8/7/S04.

[29] B. Sprenger, J. Zhang, Z. H. Lu, and L. J. Wang, "Atmospheric transfer of optical and radio frequency clock signals," Optics Letters, vol. 34, no. 7, pp. 965-967, Apr. 2009, doi: 10.1364/OL.34.000965.

[30] M. Lipka, M. Parniak, and W. Wasilewski, “Optical frequency locked loop for long-term stabilization of broad-line DFB laser frequency difference," Applied Physics B, vol. 123, no. 9, p. 238, Aug. 2017, doi: 10.1007/s00340-017-6808-6.

[31] B. Chen, K. Wu, L. Yan, J. Xie, and E. Zhang, "Stabilization of synthetic wavelength using offset-frequency locking for the measurement accuracy improvement of the laser synthetic wavelength interferometer," Optical Engineering, vol. 57, no. 3, p. 034106, Mar. 2018, doi: 10.1117/1.OE.57.3.034106. 